[37] J.-S. Jang, S.-Y. Lee, and S.-Y. Shin, "An optimization network for matrix inversion," in Neural Information Processing Systems, D. Z Anderson, Ed. College Park, MD: AIP, 1988, pp. 397-401.

[38] D. W. Tank and J. J. Hopfield, "Simple 'neural' optimization networks: An A/D converter, signal decision circuit, and a linear programming circuit," IEEE Trans. Circuits Syst., vol. 33, no. 5, pp. 533-541, May 1986.

[39] E. Liz and J. B. Ferreiro, "A note on the global stability of generalized difference equations," Appl. Math. Lett., vol. 15, no. 6, pp. 655-659, Aug. 2002.

[40] A. Halanay, Differential Equations: Stability, Oscillations, Time Lags. New York: Academic, 1966.

[41] Z. Wang, S. Lauria, J. Fang, and X. Liu, "Exponential stability of uncertain stochastic neural networks with mixed time-delays," Chaos, Solitons \& Fractals, vol. 32, no. 1, pp. 62-72, Apr. 2007.

[42] Z. Wang, Y. Liu, K. Fraser, and X. Liu, "Stochastic stability of uncertain Hopfield neural networks with discrete and distributed delays," Phys. Lett. A, vol. 354, no. 4, pp. 288-297, Jun. 2006.

[43] Z. Wang, Y. Liu, L. Yu, and X. Liu, "Exponential stability of delayed recurrent neural networks with Markovian jumping parameters," Phys. Lett. A, vol. 356, nos. 4-5, pp. 346-352, Aug. 2006.

\section{Exponential Synchronization of Linearly Coupled Neural Networks with Impulsive Disturbances}

Jianquan Lu, Daniel W. C. Ho, Senior Member, IEEE, Jinde Cao, Senior Member, IEEE, and Jürgen Kurths

\begin{abstract}
This brief investigates globally exponential synchronization for linearly coupled neural networks (NNs) with timevarying delay and impulsive disturbances. Since the impulsive effects discussed in this brief are regarded as disturbances, the impulses should not happen too frequently. The concept of average impulsive interval is used to formalize this phenomenon. By referring to an impulsive delay differential inequality, we investigate the globally exponential synchronization of linearly coupled NNs with impulsive disturbances. The derived sufficient
\end{abstract}

Manuscript received May 20, 2010; revised August 1, 2010; accepted December 11, 2010. Date of publication January 13, 2011; date of current version February 9, 2011. The work of J. Lu was supported in part by the National Natural Science Foundation of China (NSFC), under Grant 11026182, the Natural Science Foundation of Jiangsu Province of China, under Grant BK2010408, the Innovation Fund of Basic Scientific Research Operating Expenses 3207010501, and the Alexander von Humboldt Foundation of Germany. The work of D. W. C. Ho was partially supported by a grant CityU 7002561 and GRF of HKSAR (CityU 1011109). The work of J. Cao was partially supported by the National Natural Science Foundation of China under Grant 11072059 and Grant 60874088, the Specialized Research Fund for the Doctoral Program of Higher Education under Grant 20070286003, and the Natural Science Foundation of Jiangsu Province of China under Grant BK2009271. The work of J. Kurths was partially supported by Supermodeling by Combining Imperfect Models, European Union, and Evolving Complex Networks, Wissenschaftsgemeinschaft Gottfried Wilhelm Leibniz.

J. Lu is with the Department of Mathematics, Southeast University, Nanjing 210096, China. He is also with the Potsdam Institute for Climate Impact Research, Potsdam 14415, Germany (e-mail: jqluma@seu.edu.cn; jqluma@gmail.com).

D. W. C. Ho is with the Department of Mathematics, City University of Hong Kong, Hong Kong (e-mail: madaniel@cityu.edu.hk).

J. Cao is with the Department of Mathematics, Southeast University, Nanjing 210096, China (e-mail: jdcao@seu.edu.cn).

J. Kurths is with the Potsdam Institute for Climate Impact Research, Telegraphenberg, Potsdam 14415, Germany. He is also with the Department of Physics, Humboldt University Berlin, Berlin 12489, Germany, and also with the Institute for Complex Systems and Mathematical Biology, University of Aberdeen, Aberdeen AB24 3UE, U.K. (e-mail: Juergen.Kurths@pikpotsdam.de).

Color versions of one or more of the figures in this brief are available online at http://ieeexplore.ieee.org.

Digital Object Identifier 10.1109/TNN.2010.2101081 condition is closely related with the time delay, impulse strengths, average impulsive interval, and coupling structure of the systems. The obtained criterion is given in terms of an algebraic inequality which is easy to be verified, and hence our result is valid for largescale systems. The results extend and improve upon earlier work. As a numerical example, a small-world network composing of impulsive coupled chaotic delayed NN nodes is given to illustrate our theoretical result.

Index Terms-Desynchronizing impulses, globally exponential synchronization, linearly coupled neural networks.

\section{INTRODUCTION}

Recently, synchronization of complex systems and dynamical networks has attracted a great deal of attention [1]-[12]. A complex dynamical network is a large set of interconnected nodes, and each node acts a nonlinear dynamical system. From the literature, there are two common phenomena in many evolving networks: delay effects and impulsive effects [13]-[17]. Time delay is ubiquitous in the implementation of electronic networks due to the finite switching speed of amplifiers and finite signal propagation time [13], [15], [17]. Delayed chaotic neural networks (NNs) coupled with a linear coupling configuration are easy to physically implement and hence have potential applications especially in secure communications based on synchronization [18], [19]. On the other hand, the states of electronic networks and biological networks are often subject to instantaneous disturbances and experience abrupt changes at certain instants, which may be caused by switching phenomenon, frequency change, or other sudden noise, i.e., they exhibit impulsive effects [14], [16], [20], [21]. Impulsive dynamical networks, which are characterized by abrupt changes in the state differences of the systems at certain instants, have sparked the interest of many researchers for their various applications in information science, economic systems, automated control systems, etc [22]. Since delays and impulses can heavily affect the dynamical behaviors of the networks, it is necessary to investigate both the delay and impulsive effects on the synchronization of dynamical networks.

Synchronization of linearly coupled systems with impulses has also been studied in many papers [12], [14], [17], [22]-[28]. Many NNs rely on a synchronous behavior for a proper functioning, e.g., information transmission, pattern recognition, and learning [29], [30]. Hence, this brief will be devoted to studying the synchronization of coupled NNs. In most works [14], [22], [25]-[28], impulsive controllers are designed for the stabilization of complex dynamical networks. However, sometimes impulses can play a negative role for the synchronization of dynamical networks [17], [27]. In [17], it was shown that synchronization of impulsive coupled delayed dynamical networks is heavily dependent on the impulsive effects of the network connections. In [27], robust stability of complex impulsive dynamical systems was studied when the impulsive effects are destabilizing or stabilizing.

When the impulsive effects are destabilizing, the impulses should not happen too frequently [12]. In [17], [20], and [27], the lower bound of the impulsive intervals are used to represent this phenomenon, and such a formalization would lead the obtained results to be rather conservative. Hence, by referring 
to the concept of average dwell time (describing switched system) [31]-[33], a similar concept named "average impulsive interval" will be used to represent the impulse sequences in this brief [12]. The synchronization problem of dynamical networks with destabilizing impulses will be studied by using the concept of average impulsive interval. The Lyapunov method and the Kronecker product are used to derive the main results of this brief. A numerical example is also given to illustrate the effectiveness of the obtained result.

The main merits of this brief are as follows: 1) impulsive effects are destabilizing; 2) impulses occur in the process of the connected nodes' state coupling; 3) time-varying delay is only required to be bounded without any derivative constraint; 4) the concept of average-impulsive interval is used to release the constraint on the lower bound of the destabilizing impulsive interval, and hence makes the result available for a wider range of impulsive signals; and 5) our criterion is applicable to large-scale dynamical networks.

Notations: Standard notations will be used in this brief. Throughout this brief, for real symmetric matrices $X$ and $Y$, the notation $X \leq Y$ (respectively, $X<Y$ ) means that the matrix $X-Y$ is negative semidefinite (respectively, negative definite). $I_{n}$ is the identity matrix of order $n$. We use $\lambda_{\max }(\cdot)$ (respectively $\lambda_{2}(\cdot)$ ) to denote the maximum (respectively second largest) eigenvalue of a real symmetric matrix. $\mathbb{R}^{n}$ denotes the $n$-dimensional Euclidean space. $\mathbb{R}^{n \times n}$ are $n \times n$ real matrices. The superscript " $T$ " represents the transpose. $\operatorname{diag}\{\cdots\}$ stands for a block-diagonal matrix. Matrices, if not explicitly stated, are assumed to have compatible dimensions. Let $\mathbb{P C}(m)$ denote the class of piecewise right continuous function $\varphi:\left[t_{0}-\tau, t\right] \rightarrow \mathbb{R}^{m}(m \in \mathbb{N})$ with the norm defined by $\|\varphi(t)\|_{\tau}=\sup _{-\tau \leq s \leq 0}\|\varphi(t+s)\|$. For $\varphi: \mathbb{R} \rightarrow \mathbb{R}$, denote $\varphi\left(t^{+}\right)=\lim _{s \rightarrow 0^{+}} \varphi(t+s), \varphi\left(t^{-}\right)=\lim _{s \rightarrow 0^{-}} \varphi(t+s)$, $\bar{\varphi}(t)=\sup _{-\tau \leq s \leq 0}\{\varphi(t+s)\}$. The Dini derivative of $\stackrel{s \rightarrow 0^{-}}{\varphi(t)}$ is defined as $D^{+} \varphi(t)=\limsup _{s \rightarrow 0^{+}}(\varphi(t+s)-\varphi(t)) /(s)$.

\section{Model And Preliminaries}

We consider a complex system consisting of $N$ linearly coupled identical NNs. Each node is an $n$-dimensional NN composed of a linear term, a nonlinear term, a time-varying delay term, and an external input vector. The $i$ th $\mathrm{NN}$ can be described by following differential equation:

$$
\dot{x}_{i}(t)=C x_{i}(t)+B_{1} f\left(x_{i}(t)\right)+B_{2} f\left(x_{i}(t-\tau(t))\right)+I(t)
$$

where $x_{i}(t)=\left[x_{i 1}(t), x_{i 2}(t), \ldots, x_{i n}(t)\right]^{T}$ is the state vector of the $i$ th NN at time $t ; C \in \mathbb{R}^{n \times n}, B_{1} \in \mathbb{R}^{n \times n}$ and $B_{2} \in$ $\mathbb{R}^{n \times n}$ are matrices; $f\left(x_{i}(t)\right)=\left[f_{1}\left(x_{i 1}(t)\right), f_{2}\left(x_{i 2}(t)\right), \ldots\right.$, $\left.f_{n}\left(x_{i n}(t)\right)\right]^{T} ; I(t)=\left[I_{1}(t), I_{2}(t), \ldots, I_{n}(t)\right] \in \mathbb{R}^{n}$ is an external input vector; and $\tau(t)$ is a time-varying delay satisfying $0<\tau(t) \leq \tau^{*}$.

For the nonlinear functions $f_{k}(\cdot)$, we have the following assumption.

Assumption 1: Assume that $f_{k}(\cdot)(k=1,2, \ldots, n)$ are globally Lipschitz continuous functions, i.e., there exist constants $l_{k}>0(k=1,2, \ldots, n)$ such that $\left|f_{k}\left(x_{1}\right)-f_{k}\left(x_{2}\right)\right| \leq$ $l_{k}\left|x_{1}-x_{2}\right|(k=1,2, \ldots, n)$ hold for any $x_{1}, x_{2} \in \mathbb{R}$. Denote $L=\operatorname{diag}\left\{l_{1}, l_{2}, \ldots, l_{n}\right\}$ for convenience.

The dynamical behavior of linearly coupled NNs can be described by the following delayed differential equations [19], [34]:

$$
\begin{aligned}
\dot{x}_{i}(t) & =C x_{i}(t)+B_{1} f\left(x_{i}(t)\right)+B_{2} f\left(x_{i}(t-\tau(t))\right)+I(t) \\
& +c \sum_{j=1, j \neq i}^{N} a_{i j} \Gamma\left(x_{j}(t)-x_{i}(t)\right), \quad i=1, \ldots, N
\end{aligned}
$$

where $\Gamma=\operatorname{diag}\left\{\gamma_{1}, \gamma_{2}, \ldots, \gamma_{n}\right\}$ (satisfying $\gamma_{i}>0$ for $i=$ $1,2, \ldots, n)$ is the diagonal inner coupling matrix between two connected nodes $i$ and $j(i \neq j)$ at time $t$ for all $1 \leq i, j \leq N$; $c$ is the coupling strength; and $a_{i j}$ is defined as follows: if there is a connection from node $j$ to node $i(j \neq i)$, then $a_{i j}>0$; otherwise, $a_{i j}=0$. It means that the network is directed and the coupling matrix $A=\left(a_{i j}\right)_{N \times N}$ is asymmetric.

In the process of signal transmission, the coupled states $x_{j}(t)-x_{i}(t)$ between connected nodes $j$ and $i$ are suddenly changed in the form of impulses at discrete times $t_{k}$. That is, $x_{i}\left(t_{k}^{+}\right)-x_{j}\left(t_{k}^{+}\right)=S_{k} \cdot\left(x_{i}\left(t_{k}^{-}\right)-x_{j}\left(t_{k}^{-}\right)\right)$. For the sake of analytical simplification, we shall choose a constant impulse gain in the form of $S_{k}=\mu I$, where $I$ is an $n \times n$ identity matrix.

Remark 9: This simplification for the impulsive matrix is only for the simplification of the expression, and it does not cause any loss of generality in the sense of synchronization analysis. The matrix product can be used to describe the synchronization criterion if the constant matrix $S_{k}$ is used to describe the impulsive amplification.

Let $a_{i i}=-\sum_{j=1, j \neq i}^{N} a_{i j}$. Then, the impulsive dynamical system can be obtained in the following form:

$$
\left\{\begin{array}{c}
\dot{x}_{i}(t)=C x_{i}(t)+B_{1} f\left(x_{i}(t)\right)+B_{2} f\left(x_{i}(t-\tau(t))\right) \\
+I(t)+c \sum_{j=1}^{N} a_{i j} \Gamma x_{j}(t), t \geq 0, t \neq t_{k}, k \in \mathbb{N} \\
x_{j}\left(t_{k}^{+}\right)-x_{i}\left(t_{k}^{+}\right)=\mu \cdot\left(x_{j}\left(t_{k}^{-}\right)-x_{i}\left(t_{k}^{-}\right)\right), \\
\text {for } k \in \mathbb{N},(i, j) \text { satisfying } a_{i j}>0, \\
x_{i}(t)=\phi_{i}(t), \quad-\tau^{*} \leq t \leq 0
\end{array}\right.
$$

where $\left\{t_{1}, t_{2}, t_{3}, \ldots\right\}$ is a sequence of strictly increasing impulsive moments, and $|\mu|>1$ represents the strength of impulsive disturbances between the coupling of the connected nodes. Here, $A=\left(a_{i j}\right)_{N \times N}$ is a Laplacian matrix of the corresponding network [35]. We always assume that $x_{i}(t)$ is right continuous at $t=t_{k}$, i.e., $x\left(t_{k}\right)=x\left(t_{k}^{+}\right)$. Therefore, the solutions of (3) are piecewise right-hand continuous functions with discontinuities at $t=t_{k}$ for $k \in \mathbb{N}$.

By referring to the concept of average dwell time [31], [32], we propose a new concept named average impulsive interval to describe the impulse signal in [12]. This concept has been utilized for the derivation of a unified synchronization criterion of dynamical networks in [12]. Since $|\mu|>1$, which means that the impulses can potentially destroy the synchronization, we need to require that they do not happen too frequently. Definition 2 is then given to enforce an upper bound on the number of impulsive times.

Definition 2 ([12] average impulsive interval): The average impulsive interval of the impulsive sequence $\zeta=$ 
$\left\{t_{1}, t_{2}, \ldots\right\}$ is said to be not less than $T_{a}$ if there exist a positive integer $N_{0}$ and a positive number $T_{a}$ such that

$$
N_{\zeta}(T, t) \leq \frac{T-t}{T_{a}}+N_{0} \quad \forall T \geq t \geq 0
$$

where $N_{\zeta}(T, t)$ denotes the number of impulsive times of the impulsive sequence $\zeta$ on the interval $(t, T)$.

Remark 10: When $N_{0}=1$, the consecutive impulses must be separated by at least $T_{a}$ units of time. When $N_{0} \geq 2$, for arbitrarily small $\epsilon$ and any $T_{a}>0$, many impulsive sequences $\left\{t_{1}, t_{2}, \ldots\right\}$ can be constructed such that the lower bound of the impulsive intervals are less than $\epsilon$ and simultaneously the average impulsive intervals are not less than $T_{a}$. Let $N_{0} \geq 2$, one simple example being $\zeta^{*}=\left\{t_{0}+2 T_{a}, t_{0}+2 T_{a}+\epsilon, t_{0}+\right.$ $\left.4 T_{a}, t_{0}+4 T_{a}+\epsilon, \ldots\right\}$. For the impulsive sequence $\zeta^{*}$, the lower bound of the impulsive interval is $\epsilon$. Since the lower bound is used to represent the frequency of the impulsive sequence in [17], [20] and [27], the results obtained in [17], [20] and [27] are not available for the impulsive sequence $\zeta^{*}$ with sufficiently small $\epsilon$.

Remark 11: The idea behind this concept is as follows. High-density impulses (such as " $t_{0}+2 T_{a}, t_{0}+2 T_{a}+\epsilon$ ") are allowed to happen in a certain interval, and low-density impulses (such as " $t_{0}+2 T_{a}+\epsilon, t_{0}+4 T_{a}$ ") should follow for compensation.

Definition 3 ([36]): The impulsive dynamical network (3) is said to be globally exponentially synchronized if there exist an $\eta>0, T>0$, and $M_{0}>0$ such that for any initial values $\phi_{i}(s)(i=1,2, \ldots, N)$

$$
\left\|x_{i}(t)-x_{j}(t)\right\| \leq M_{0} e^{-\eta t}
$$

hold for all $t>T$, and for any $i, j=1,2, \ldots, N$.

Definition 4 ([37]): For an irreducible square matrix $A$ with nonnegative off-diagonal elements, the functions $\alpha(A)$ and $\beta(A)$ are defined as follows: decompose $A$ uniquely as $A=L_{A}+D_{A}$, where $L_{A}$ is a zero row sum matrix and $D_{A} \leq 0$ is a diagonal matrix. Let $\xi=\left(\xi_{1}, \xi_{2}, \ldots, \xi_{N}\right)^{T}$ be the unique positive vector such that $\xi^{T} L_{A}=0$ and $\sum_{i=1}^{N} \xi_{i}=1$. The existence of such a positive vector $\xi$ can be guaranteed by the following Lemma 2 . Let $\Xi=$ $\operatorname{diag}(\xi)$. Then $\alpha(A)=\lambda_{2}\left(\Xi A+A^{T} \Xi\right)$ and $\beta(A)=$ $\lambda_{\max }\left(\Xi A+A^{T} \Xi\right)$.

Lemma 1 ([37]): Let $A$ be an irreducible matrix with nonnegative off-diagonal elements and nonnegative row sums with the decomposition $A=L_{A}+D_{A}$ as shown in Definition 4. Then $\alpha(A)<0, \beta(A) \leq 0$. Furthermore, $\beta(A)<0$ if and only if $D_{A} \neq 0$.

Lemma 2: For any vectors $x, y \in R^{n}$, we have $2 x^{T} y \leq$ $x^{T} x+y^{T} y$.

Lemma 3 ([19], [38] (Perron-Frobenius Theorem)): For an irreducible matrix $A$ with nonnegative off-diagonal elements, which satisfies the diffusive coupling condition $a_{i i}=$ $-\sum_{j=1, j \neq i}^{N} a_{i j}$, we have the following propositions.

1) If $\lambda$ is an eigenvalue of $A$ and $\lambda \neq 0$, then $\operatorname{Re}(\lambda)<0$.

2) $A$ has an eigenvalue 0 with multiplicity 1 and the right eigenvector $[1,1, \ldots, 1]^{T}$.

3) Suppose that $\xi=\left[\xi_{1}, \xi_{2}, \ldots, \xi_{N}\right]^{T} \in \mathbb{R}^{N}$ satisfying $\sum_{i=1}^{N} \xi_{i}=1$ is the normalized left eigenvector of $A$ corresponding to eigenvalue 0 . Then, $\xi_{i}>0$ hold for all $i=1,2, \ldots, N$. Furthermore, if $A$ is symmetric, then we have $\xi_{i}=1 / N$ for $i=1,2, \ldots, N$.

Lemma 4 ([20]): Suppose $p>q \geq 0$ and $u(t)$ satisfies the scalar impulsive differential inequality

$$
\begin{cases}D^{+} u(t) \leq-p u(t)+q \bar{u}(t), & t \neq t_{k}, t \geq t_{0} \\ u\left(t_{k}^{+}\right) \leq \rho_{k} u\left(t_{k}^{-}\right), & k \in \mathbb{N} \\ u(t)=\phi(t), & t \in\left[t_{0}-\tau, t_{0}\right]\end{cases}
$$

where $u(t)$ is continuous at $t \neq t_{k}, t \geq t_{0}, u\left(t_{k}\right)=u\left(t_{k}^{+}\right)$, and $u\left(t_{k}^{-}\right)$exists, $\phi \in \mathbb{P} \mathbb{C}(1)$, and $\bar{u}(t)=\sup _{-\tau \leq s \leq 0}\{u(t+s)\}$. Then

$$
u(t) \leq\left\|\phi\left(t_{0}\right)\right\|_{\tau} \cdot\left(\prod_{t_{0}<t_{k} \leq t} \rho_{k}\right) \cdot e^{-\lambda\left(t-t_{0}\right)}, t \geq t_{0}
$$

where $\lambda>0$ is the unique solution of the equation $\lambda-p+$ $q e^{\lambda \tau}=0$.

Remark 12: The result of Lemma 4 extends the famous Halanay differential inequality [39] to impulsive delay differential systems, and will be used for the proof of the following Proposition 1.

Proposition 1: Suppose $p>q \geq 0$ and $u(t)$ satisfies the scalar impulsive differential inequality

$$
\begin{cases}D^{+} u(t) \leq-p u(t)+q \bar{u}(t), & t \neq t_{k}, t \geq t_{0} \\ u\left(t_{k}^{+}\right) \leq \rho u\left(t_{k}^{-}\right), & k \in \mathbb{N} \\ u(t)=\phi(t), & t \in\left[t_{0}-\tau, t_{0}\right]\end{cases}
$$

where $u(t)$ is continuous at $t \neq t_{k}, t \geq t_{0}, u\left(t_{k}\right)=u\left(t_{k}^{+}\right)$, $u\left(t_{k}^{-}\right)$exists, $\phi \in \mathbb{P} \mathbb{C}(1),|\rho|>1$, and the average impulsive interval of the impulsive sequence $\zeta=\left\{t_{1}, t_{2}, \ldots\right\}$ is not less than $T_{a}$. Then there exists a constant $M_{0}$ such that

$$
u(t) \leq M_{0} e^{-\left(\lambda-\frac{\ln |\rho|}{T_{a}}\right)\left(t-t_{0}\right)}, \quad t \geq t_{0}
$$

where $\lambda>0$ is the unique solution of the equation $\lambda-p+$ $q e^{\lambda \tau}=0$.

Proof: See Appendix.

Remark 13: Proposition 1 plays an important role in the synchronization analysis of dynamical networks with impulsive disturbances in this brief, because it shows the utilization of the concept of average impulsive interval.

\section{SYNCHRONIZATION ANALYSIS}

In this section, globally exponential synchronization of linearly coupled NNs with impulsive disturbances will be studied. Let $x(t)=\left(x_{1}^{T}(t), x_{2}^{T}(t), \ldots, x_{N}^{T}(t)\right)^{T}, F(x(t))=$ $\left(f^{T}\left(x_{1}(t)\right), f^{T}\left(x_{2}(t)\right), \ldots, f^{T}\left(x_{N}(t)\right)\right)^{T}, \quad$ and $\quad \mathbf{I}(t)=$ $\left(I^{T}(t), I^{T}(t), \ldots, I^{T}(t)\right)^{T}$, then the impulsive dynamical system (3) can be rewritten in the following Kroneckerproduct form:

$$
\left\{\begin{array}{c}
\dot{x}(t)=\left(I_{N} \otimes C\right) x(t)+\left(I_{N} \otimes B_{1}\right) F(x(t)) \\
+\left(I_{N} \otimes B_{2}\right) F(x(t-\tau(t)))+\mathbf{I}(t) \\
\quad+c(A \otimes \Gamma) x(t), t \geq 0, t \neq t_{k}, k \in \mathbb{N} \\
x_{j}\left(t_{k}^{+}\right)-x_{i}\left(t_{k}^{+}\right)=\mu \cdot\left(x_{j}\left(t_{k}^{-}\right)-x_{i}\left(t_{k}^{-}\right)\right) \\
\text {for } k \in \mathbb{N},(i, j) \text { satisfying } a_{i j}>0 \\
x_{i}(t)=\phi_{i}(t), \quad-\tau^{*} \leq t \leq 0 .
\end{array}\right.
$$


Suppose that $\xi=\left(\xi_{1}, \xi_{2}, \ldots, \xi_{N}\right)^{T}$ is the normalized left eigenvector of the configuration coupling matrix $A$ with respect to the eigenvalue 0 satisfying $\sum_{i=1}^{N} \xi_{i}=1$. Since the coupling configuration matrix $A=\left(a_{i j}\right)_{N \times N}$ is irreducible, according to the Lemma 3 , we can conclude that $\xi_{i}>0$ for $i=1,2, \ldots, N$. Let $\Xi=\operatorname{diag}\left\{\xi_{1}, \xi_{2}, \ldots, \xi_{N}\right\}>0$, and $W \triangleq\left(w_{i j}\right)_{N \times N}=\Xi-\xi \xi^{T}$.

Theorem 2: Consider the linearly coupled NNs (9) with impulsive disturbances and the asymmetric irreducible coupling matrix $A$. Let $\gamma=-\alpha(A) / \lambda_{\max }(W), p=-\lambda_{\max }(2 C-$ $\left.c \gamma \Gamma+B_{1} B_{1}^{T}+L^{T} L+B_{2} B_{2}^{T}\right)$, and $q=\lambda_{\max }\left(L^{T} L\right)$, where $L$ is the Lipschitz matrix given in Assumption 1, and $\alpha(A)$ is defined in Definition 4. Suppose that Assumption 1 holds, and then the average impulsive interval of the impulsive sequence $\zeta=\left\{t_{1}, t_{2}, \ldots\right\}$ is not less than $T_{a}$. Then, the linearly coupled NNs (9) with impulsive disturbances will be globally exponentially synchronized with the convergence rate $\lambda-(2 \ln |\mu|) /\left(T_{a}\right)$ if

$$
p>q \text { and } \lambda-\frac{2 \ln |\mu|}{T_{a}}>0
$$

where $\lambda>0$ is the unique solution of the equation $\lambda-p+$ $q e^{\lambda \tau^{*}}=0$.

Proof: Let $V(t)=x^{T}(t)\left(W \otimes I_{n}\right) x(t)$. By a detailed calculation, we get that $V(t)=(1 / 2) \sum_{i=1}^{N} \sum_{j=1, j \neq i}^{N}-w_{i j}\left(x_{i}-\right.$ $\left.x_{j}\right)^{T}\left(x_{i}-x_{j}\right)$. It can be observed that $V(t)$ is a smooth and positive semidefinite function since $-w_{i j}=\xi_{i} \xi_{j}>0$. In fact, the function $V(t)$ vanishes at the synchronization manifold $\mathcal{M}=\left\{x_{1}(t)=x_{2}(t)=\ldots=x_{N}(t)\right\}$. The derivative of $V(t)$ along the trajectories of the systems (9) can be obtained as follows:

$$
\begin{aligned}
D^{+} V(x) & \\
= & 2 x^{T}(t)(W \otimes C) x(t)+2 x^{T}(t)\left(W \otimes B_{1}\right) F(x(t)) \\
& +2 x^{T}(t)\left(W \otimes B_{2}\right) F(x(t-\tau(t))) \\
& +2 x^{T}(t)\left(W \otimes I_{n}\right) \mathbf{I}(t)+2 c x^{T}(t)(W A \otimes \Gamma) x(t), \\
& t \in\left(t_{k-1}, t_{k}\right], \quad k \in \mathbb{N} .
\end{aligned}
$$

From Assumption 1, we have

$$
\begin{aligned}
& \left(f\left(x_{i}\right)-f\left(x_{j}\right)\right)^{T}\left(f\left(x_{i}\right)-f\left(x_{j}\right)\right) \\
& \leq\left(x_{i}-x_{j}\right)^{T} L^{T} L\left(x_{i}-x_{j}\right) .
\end{aligned}
$$

Noting that $W A=\left(\Xi-\xi \xi^{T}\right) A=\Xi A-\xi\left(\xi^{T} A\right)=\Xi A$ and $\left(W \otimes I_{n}\right) \mathbf{I}(t)=0$, it follows that

$$
\begin{aligned}
& D^{+} V(x) \\
& \leq-\sum_{i=1}^{N} \sum_{j=1, j \neq i}^{N} w_{i j}\left\{( x _ { i } - x _ { j } ) ^ { T } \left(C-\frac{1}{2} c \gamma \Gamma+\frac{1}{2} B_{1} B_{1}^{T}\right.\right. \\
& \left.+\frac{1}{2} L^{T} L+\frac{1}{2} B_{2} B_{2}^{T}\right)\left(x_{i}-x_{j}\right)+\frac{1}{2}\left[x_{i}(t-\tau(t))\right. \\
& \left.\left.-x_{j}(t-\tau(t))\right]^{T} L^{T} L\left[x_{i}(t-\tau(t))-x_{j}(t-\tau(t))\right]\right\} \\
& +c x^{T}(t)\left[\left(\Xi A+A^{T} \Xi\right) \otimes \Gamma+W \otimes \gamma \Gamma\right] x(t), \\
& \quad t \in\left(t_{k-1}, t_{k}\right], \quad k \in \mathbb{N}
\end{aligned}
$$

where $w_{i j}=-\xi_{i} \xi_{j}<0$ for $i \neq j$, inequality (12), and Lemma 2 are used.
Since $p=-\lambda_{\max }\left(2 C-c \gamma \Gamma+B_{1} B_{1}^{T}+L^{T} L+B_{2} B_{2}^{T}\right)$ and $q=\lambda_{\max }\left(L^{T} L\right)$, it follows from (13) that

$$
\begin{aligned}
& D^{+} V(x) \\
& \leq-p \sum_{i=1}^{N} \sum_{j=1, j \neq i}^{N}\left[-\frac{1}{2} w_{i j}\left(x_{i}-x_{j}\right)^{T}\left(x_{i}-x_{j}\right)\right] \\
&+q \sum_{i=1}^{N} \sum_{j=1, j \neq i}^{N}-\frac{1}{2} w_{i j}\left[\left(x_{i}(t-\tau(t))-x_{j}(t-\tau(t))\right)^{T}\right. \\
&\left.\times\left(x_{i}(t-\tau(t))-x_{j}(t-\tau(t))\right)\right] \\
&+c x^{T}(t)\left[\left(\Xi A+A^{T} \Xi\right) \otimes \Gamma+W \otimes \gamma \Gamma\right] x(t) \\
&=-p V(t)+q V(t-\tau(t)) \\
&+c x^{T}(t)\left[\left(\Xi A+A^{T} \Xi\right) \otimes \Gamma+W \otimes \gamma \Gamma\right] x(t) \\
& \leq-p V(t)+q \bar{V}(t)+c x^{T}(t)\left[\left(\Xi A+A^{T} \Xi\right) \otimes \Gamma\right. \\
&+W \otimes \gamma \Gamma] x(t), \quad t \in\left(t_{k-1}, t_{k}\right], \quad k \in \mathbb{N}
\end{aligned}
$$

where $\bar{V}(t)=\sup _{-\tau^{*} \leq s \leq 0}\{V(t+s)\}$.

Let $\tilde{A}=\Xi A+A^{T} \Xi$. From the Perron-Frobenius theorem [38], the eigenvalues of the matrix $\tilde{A}$ can be arranged as follows: $0=\lambda_{1}(\tilde{A})>\lambda_{2}(\tilde{A}) \geq \lambda_{3}(\tilde{A}) \geq \ldots \geq \lambda_{N}(\tilde{A})$, where $\lambda_{2}(\tilde{A})=\alpha(A)$ by Definition 4 .

From matrix decomposition theory [38], there exists a unitary matrix $U$ such that $\tilde{A}=U \Lambda U^{T}$, where $\Lambda=$ $\operatorname{diag}\left\{0, \lambda_{2}(\tilde{A}), \ldots, \lambda_{N}(\tilde{A})\right\}$, and $U=\left[u_{1}, u_{2}, \ldots, u_{N}\right]$ with $u_{1}=((1 / \sqrt{N}),(1 / \sqrt{N}), \ldots,(1 / \sqrt{N}))^{T}$.

Let $y(t)=\left(U^{T} \otimes I_{n}\right) x(t)$, then one has $x(t)=(U \otimes$ $\left.I_{n}\right) y(t)$. Let $y_{i}(t) \in \mathbb{R}^{n}(i=1,2, \ldots, N)$ be such that $y(t)=$ $\left[y_{1}^{T}(t), y_{2}^{T}(t), \ldots, y_{N}^{T}(t)\right]^{T}$, and it follows that

$$
\begin{aligned}
x^{T}(t)[(\Xi A & \left.\left.+A^{T} \Xi\right) \otimes \Gamma\right] x(t) \\
& =y^{T}(t)\left(U^{T} \otimes I_{n}\right)(\tilde{A} \otimes \Gamma)\left(U \otimes I_{n}\right) y(t) \\
& =\sum_{i=2}^{N} \lambda_{i}(\tilde{A}) y_{i}^{T}(t) \Gamma y_{i}(t) \\
& \leq \lambda_{2}(\tilde{A}) \sum_{i=2}^{N} y_{i}^{T}(t) \Gamma y_{i}(t) \\
& =\alpha(A) \sum_{i=2}^{N} y_{i}^{T}(t) \Gamma y_{i}(t) .
\end{aligned}
$$

From the construction of matrix $W$, it can be observed that $W$ is a zero row sum irreducible symmetric matrix with negative off-diagonal elements. Hence it follows that $\lambda_{\max }(W)>0$. Moreover, $W \cdot u_{1}=(0,0, \ldots, 0)^{T}:=O_{n} \in \mathbb{R}^{N}$. Then we get $U^{T} W U=\left[\begin{array}{cc}0 & O_{n}^{T} \\ O_{n} & \tilde{U}^{T} W \tilde{U}\end{array}\right]$, where $\tilde{U}=\left[u_{2}, u_{3}, \ldots, u_{N}\right]$ satisfying $\tilde{U}^{T} \tilde{U}=I_{N-1}$. Hence, one has

$$
\begin{aligned}
x^{T}(t)(W \otimes & \gamma \Gamma) x(t) \\
& =\gamma y^{T}(t)\left(U^{T} W U \otimes \Gamma\right) y(t) \\
& =\gamma \tilde{y}^{T}(t)\left(\tilde{U}^{T} W \tilde{U} \otimes \Gamma\right) \tilde{y}(t) \\
& \leq \gamma \lambda_{\max }(W) \tilde{y}^{T}(t)\left(\tilde{U}^{T} \tilde{U} \otimes \Gamma\right) \tilde{y}(t) \\
& =\gamma \lambda_{\max }(W) \sum_{i=2}^{N} y_{i}^{T}(t) \Gamma y_{i}(t)
\end{aligned}
$$

where $\tilde{y}(t)=\left[y_{2}^{T}(t), \ldots, y_{N}^{T}(t)\right]^{T}$. 
It follows from (15), (16), and the equality $\gamma=$ $-\alpha(A) / 2 \lambda_{\max }(W)$ that

$$
\begin{aligned}
c x^{T}(t) & {\left[\left(\Xi A+A^{T} \Xi\right) \otimes \Gamma+W \otimes \gamma \Gamma\right] } \\
= & c\left(\alpha(A)+\gamma \lambda_{\max }(W)\right) \sum_{i=2}^{N} y_{i}^{T}(t) \Gamma y_{i}(t) \\
& =0 .
\end{aligned}
$$

Recalling (14), one gets

$$
D^{+} V(x) \leq-p V(t)+q \bar{V}(t), t \in\left(t_{k-1}, t_{k}\right], k \in \mathbb{N} .
$$

Since $A$ is irreducible, for any pair of suffixes $i$ and $j$ $(i \neq j)$, there exist suffixes $s_{1}, s_{2}, \ldots, s_{m}$ such that $a_{j s_{1}}>0$, $a_{s_{1} s_{2}}>0, \ldots$, and $a_{s_{m} i}>0$. From (9), we have $x_{j}\left(t_{k}^{+}\right)-$ $x_{i}\left(t_{k}^{+}\right)=\mu \cdot\left(x_{j}\left(t_{k}^{-}\right)-x_{i}\left(t_{k}^{-}\right)\right), k \in \mathbb{N}$, for each pair of $(i, j)$ satisfying $a_{i j}>0$. Then, for any pair of suffixes $i$ and $j$, we get

$$
\begin{aligned}
x_{j}\left(t_{k}^{+}\right)- & x_{i}\left(t_{k}^{+}\right) \\
= & \left(x_{j}\left(t_{k}^{+}\right)-x_{s_{1}}\left(t_{k}^{+}\right)\right)+\left(x_{s_{1}}\left(t_{k}^{+}\right)-x_{s_{2}}\left(t_{k}^{+}\right)\right) \\
& +\cdots+\left(x_{s_{m}}\left(t_{k}^{+}\right)-x_{i}\left(t_{k}^{+}\right)\right) \\
= & \mu \cdot\left(x_{j}\left(t_{k}^{-}\right)-x_{s_{1}}\left(t_{k}^{-}\right)\right)+\mu \cdot\left(x_{s_{1}}\left(t_{k}^{-}\right)-x_{s_{2}}\left(t_{k}^{-}\right)\right) \\
& +\cdots+\mu \cdot\left(x_{s_{m}}\left(t_{k}^{-}\right)-x_{i}\left(t_{k}^{-}\right)\right) \\
= & \mu \cdot\left(x_{j}\left(t_{k}^{-}\right)-x_{i}\left(t_{k}^{-}\right)\right) .
\end{aligned}
$$

Hence, for $t=t_{k}, k \in \mathbb{N}$, we get

$$
\begin{aligned}
& V\left(t_{k}^{+}\right) \\
& =\frac{1}{2} \sum_{i=1}^{N} \sum_{\substack{j=1 \\
j \neq i}}^{N}-w_{i j}\left(x_{i}\left(t_{k}^{+}\right)-x_{j}\left(t_{k}^{+}\right)\right)^{T}\left(x_{i}\left(t_{k}^{+}\right)-x_{j}\left(t_{k}^{+}\right)\right) \\
& =\frac{\mu^{2}}{2} \sum_{i=1}^{N} \sum_{\substack{j=1 \\
j \neq i}}^{N}-w_{i j}\left(x_{i}\left(t_{k}^{-}\right)-x_{j}\left(t_{k}^{-}\right)\right)^{T}\left(x_{i}\left(t_{k}^{-}\right)-x_{j}\left(t_{k}^{-}\right)\right) \\
& =\mu^{2} V\left(t_{k}^{-}\right) .
\end{aligned}
$$

Employing Proposition 1 and from (18) and (20), we can conclude that there exists constant $M_{0}$ such that

$$
V(t) \leq M_{0} e^{-\left(\lambda-\frac{2 \ln |\mu|}{T_{a}}\right)(t)}, \quad t \geq 0
$$

where $\lambda>0$ is the unique solution of the equation $\lambda-p+q e^{\lambda \tau^{*}}=0$. Hence, we have $(1 / 2) \xi_{i} \xi_{j}\left\|x_{i}(t)-x_{j}(t)\right\|^{2} \leq$ $V(t) \leq M_{0} e^{-\left(\lambda-(2 \ln |\mu|) /\left(T_{a}\right)\right)(t)}$. Since $\lambda-(2 \ln |\mu|) /\left(T_{a}\right)>0$, globally exponential synchronization of linearly coupled NNs (9) with impulsive disturbances is achieved according to Definition 3. The proof is completed.

Remark 14: In this brief, we require this network to be strongly connected, which means that there is a path from each node in the network to every other node. By referring to the concept of $\beta(A)$ in Definition 4, the results in Theorem 2 and techniques in [11], the results can be extended to the case of reducible coupling (containing rooted spanning tree [37]), which means that the network is not required to be strongly connected. Details will not be discussed here due limitations of space. (a)

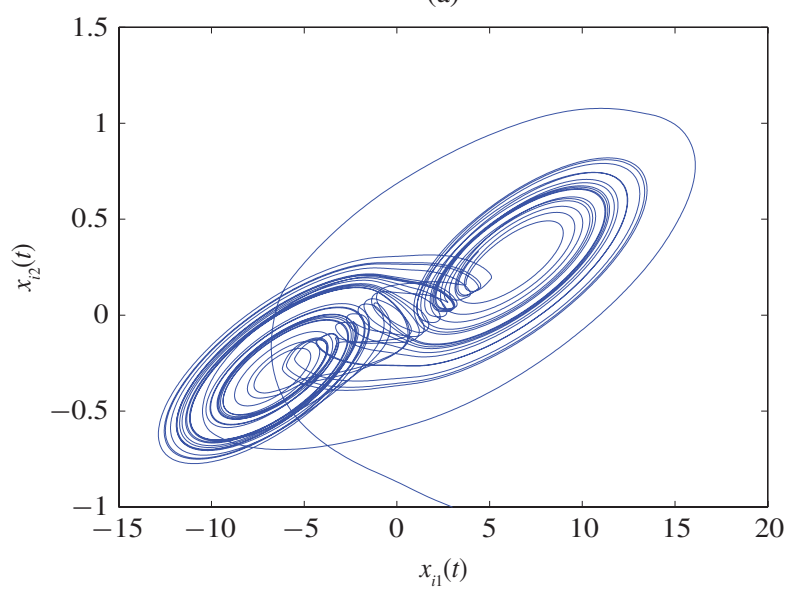

Fig. 1. Chaotic attractor of system (22).

\section{NUMERICAL EXAMPLE}

As an application of the theoretical result, the chaos synchronization problem of a typical small-world network with $100 \mathrm{NNs}$ is discussed in this section. A chaotic NN is chosen as the isolated node of the network, which can be described by the following [40]:

$\dot{x}(t)=-C x(t)+B_{1} f(x(t))+B_{2} f(x(t-\tau(t)))+I(t)$

with $C=\left(\begin{array}{ll}1 & 0 \\ 0 & 1\end{array}\right), A=\left(\begin{array}{cc}2.0 & -0.11 \\ -5.0 & 3.2\end{array}\right), B=$ $\left(\begin{array}{cc}-1.6 & -0.1 \\ -0.18 & -2.4\end{array}\right), I=\left(\begin{array}{l}0 \\ 0\end{array}\right)$, and $\tau(t)=\left(e^{t} / 1+e^{t}\right)$, where $x(t)=\left(x_{1}(t), x_{2}(t)\right)^{T}$ is the state vector of the network, and $f(x(t))=g(x(t))=\left(\tanh \left(x_{1}\right), \tanh \left(x_{2}\right)\right)^{T}$. Then the Lipschitz constants can be obtained as $l_{1}=l_{2}=1$. The single NN model (22) has a chaotic attractor as shown in Fig. 1 with the initial values $x_{1}(s)=0.2, x_{2}(s)=0.5, \forall s \in[-1,0]$.

We consider an NW small-world network with $100 \mathrm{dy}$ namical nodes [41]. The NW small-world model algorithm is presented as follows. 1) Begin with a nearest neighbor coupled network consisting of $N$ nodes arranged in a ring, where each node $i$ is adjacent to its neighbor nodes, $i=1,2, \ldots,(k / 2)$, with $k$ being even. 2) Add a connection between each pair of nodes with probability $p$. In this example, the parameters are set as $N=100, k=2$, and $p=0.04$. Then small-world network can be generated with the coupling matrix $A$. In this example, we generate 100 small-world networks randomly. By calculation, the quantities $\gamma=-\alpha(A) / \lambda_{\max }(W)$ of these 100 networks belong to $[2.7146,4.7901]$. From Theorem 2, we can observe that the remaining 99 small-world networks can be synchronized if the small-world network with minimum $\gamma=-\alpha(A) / \lambda_{\max }(W)=2.7146$, in which $\alpha(A)=-0.0785$ and $\lambda_{\max }(W)=0.0289$ can be synchronized. Hence, the small-world network with $\gamma=2.7146$ is selected for simulation in this example.

Let $c=8$ and $\Gamma=\operatorname{diag}\{5,5\}$. By a simple computation, we obtain $p=65.9471, q=1$ and $\tau^{*}=1$. The unique solution of the equation $\lambda-p+q e^{\lambda \tau^{*}}=0$ is $\lambda=4.1243$. Let the impulsive strength $\mu$ be $\mu=1.2$. According to 


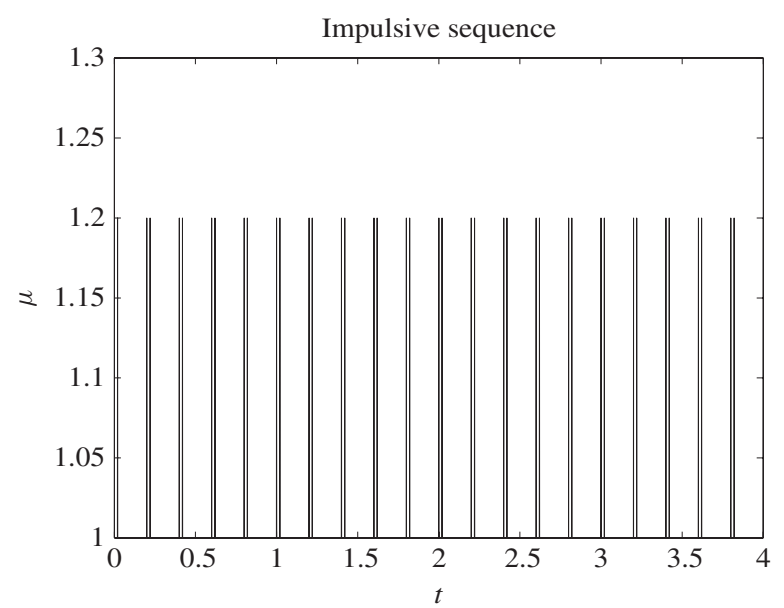

Fig. 2. Impulsive sequence $\bar{\zeta}$ with $\epsilon=0.02$ in (23).

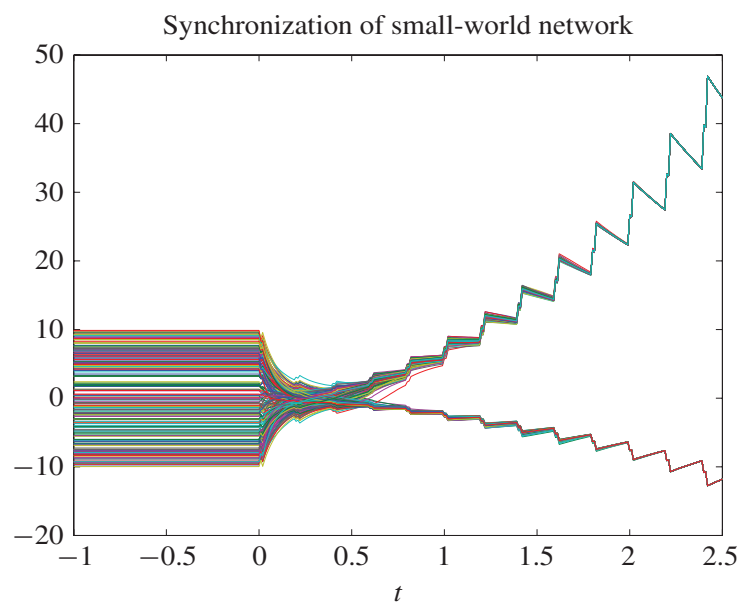

Fig. 3. (Color online.) State variables $x_{1}(t)$ and $x_{2}(t)$ of small-world coupled dynamical network with impulsive disturbances.

Theorem 2, it can be concluded that the complex dynamical network with impulsive disturbance can be synchronized if the average impulsive interval $T_{a}$ of the impulsive sequence is not less than 0.0884. Take the impulsive sequence as

$$
\bar{\zeta}=\{\epsilon, 0.2,0.2+\epsilon, 0.4,0.4+\epsilon, 0.6, \ldots\}
$$

with $\epsilon<0.1$. According to Definition 2, the average impulsive interval of $\bar{\zeta}$ is not less than 0.1 by taking $N_{0}=2$. Hence, according to Theorem 2, one can conclude that synchronization of the dynamical network with such impulsive disturbances can be achieved. Let $\epsilon=0.02$, Fig. 2 represents the impulsive sequence $\bar{\zeta}$, and the synchronization process of the smallworld coupled dynamical network is plotted in Fig. 3. All initial conditions in Fig. 3 are randomly selected from $[-10,10]$.

Remark 15: For the impulsive sequence $\bar{\zeta}$, the lower bound of the impulsive intervals is $\epsilon$. Since the lower bound of the impulsive intervals is used for a corresponding analysis in [17], [20], and [27], the results obtained in [17], [20], and [27] are not applicable for systems with such kind of impulsive sequence $\bar{\zeta}$ when $\epsilon$ is sufficiently small.

\section{CONCLUSION}

In this brief, globally exponential synchronization of complex dynamical networks with impulsive disturbances was studied. Since the impulsive disturbances are destabilizing, the impulses should not happen too frequently. The concept of average impulsive interval was used to describe this phenomenon. It was shown that the utilization of this concept could make the results much less conservative. Based on this concept, a less conservative criterion, which is represented by an algebraic inequality and hence applicable for large-scale systems, was derived for the synchronization analysis of a dynamical network with impulsive disturbances. A numerical example with a small-world coupling was also given to illustrate our theoretical results.

In this brief, we have studied the synchronization problem of dynamical networks with desynchronizing impulses. Naturally, one may ask: when would the synchronization of networks be destroyed by the impulses? Hence, in the future, we will study desynchronization criteria for the problem under how large impulsive strength the dynamical network would be desynchronized.

\section{APPENDIX \\ PROOF OF PROPOSITION 1}

Proof: From Lemma 4, we obtain

$$
u(t) \leq\left\|\phi\left(t_{0}\right)\right\|_{\tau} \cdot|\rho|^{N_{\zeta}\left(t, t_{0}\right)} \cdot e^{-\lambda\left(t-t_{0}\right)}, \quad t \geq t_{0}
$$

where $N_{\zeta}(t, 0)$ is the number of impulsive times of the impulsive sequence $\zeta$ on the interval $(0, t)$. Since $|\rho|>1$, it follows from (24) that

$$
\begin{aligned}
u(t) & \leq\left\|\phi\left(t_{0}\right)\right\|_{\tau} \cdot|\rho|^{\left(\frac{t-t_{0}}{T_{a}}+N_{0}\right)} \cdot e^{-\lambda\left(t-t_{0}\right)} \\
& =|\rho|^{N_{0}} \cdot\left\|\phi\left(t_{0}\right)\right\|_{\tau} \cdot e^{\frac{\ln |\rho|}{T_{a}}\left(t-t_{0}\right)} \cdot e^{-\lambda\left(t-t_{0}\right)} \\
& =|\rho|^{N_{0}} \cdot\left\|\phi\left(t_{0}\right)\right\|_{\tau} \cdot e^{-\left(\lambda-\frac{\ln |\rho|}{T_{a}}\right)\left(t-t_{0}\right)} .
\end{aligned}
$$

Hence, we conclude that there exists a constant $M_{0}=$ $|\rho|^{N_{0}} \cdot\left\|\phi\left(t_{0}\right)\right\|_{\tau}$ such that $u(t) \leq M_{0} e^{-\left(\lambda-\left(\ln |\rho| / T_{a}\right)\right)\left(t-t_{0}\right)}$ for $t \geq t_{0}$.

\section{REFERENCES}

[1] A. Arenas, A. Díaz-Guilera, J. Kurths, Y. Moreno, and C. Zhou, "Synchronization in complex networks," Phys. Rep., vol. 469, no. 3, pp. 93-153, Dec. 2008.

[2] X. F. Wang and G. Chen, "Synchronization in scale-free dynamical networks: Robustness and fragility," IEEE Trans. Circuits Syst.-I: Fundam. Theory Appl., vol. 49, no. 1, pp. 54-62, Jan. 2002.

[3] J. Lü and G. Chen, "A time-varying complex dynamical network model and its controlled synchronization criteria," IEEE Trans. Autom. Control, vol. 50, no. 6, pp. 841-846, Jun. 2005.

[4] J. Lu, D. W. C. Ho, and J. Cao, "Synchronization in an array of nonlinearly coupled chaotic neural networks with delay coupling," Int. J. Bifur. Chaos, vol. 18, no. 10, pp. 3101-3111, Dec. 2008.

[5] J. Lu and D. W. C. Ho, "Local and global synchronization in general complex dynamical networks with delay coupling," Chaos, Solitons Fractals, vol. 37, no. 5, pp. 1497-1510, Sep. 2008.

[6] H. Gao, J. Lam, and G. Chen, "New criteria for synchronization stability of general complex dynamical networks with coupling delays," Phys. Lett. A, vol. 360, no. 2, pp. 263-273, Dec. 2006.

[7] Z. Wang, Y. Wang, and Y. Liu, "Global synchronization for discrete-time stochastic complex networks with randomly occurred nonlinearities and mixed time delays," IEEE Trans. Neural Netw., vol. 21, no. 1, pp. 11-25, Jan. 2010. 
[8] J. Liang, Z. Wang, Y. Liu, and X. Liu, "Robust synchronization of an array of coupled stochastic discrete-time delayed neural networks," IEEE Trans. Neural Netw., vol. 19, no. 11, pp. 1910-1921, Nov. 2008.

[9] J. Lu, D. W. C. Ho, and Z. Wang, "Pinning stabilization of linearly coupled stochastic neural networks via minimum number of controllers," IEEE Trans. Neural Netw., vol. 20, no. 10, pp. 1617-1629, Oct. 2009 .

[10] J. Lu, D. W. C. Ho, and L. Wu, "Exponential stabilization of switched stochastic dynamical networks," Nonlinearity, vol. 22, no. 4, pp. 889911, 2009.

[11] J. Lu and D. W. C. Ho, "Globally exponential synchronization and synchronizability for general dynamical networks," IEEE Trans. Syst., Man, Cybern., Part B, vol. 40, no. 2, pp. 350-361, Apr. 2010.

[12] J. Lu, D. W. C. Ho, and J. Cao, "A unified synchronization criterion for impulsive dynamical networks," Automatica, vol. 46, no. 7, pp. 12151221, Jul. 2010 .

[13] C. Li and G. Chen, "Synchronization in general complex dynamical networks with coupling delays," Phys. A: Stat. Mech. Appl., vol. 343, pp. 263-278, Nov. 2004.

[14] B. Liu, X. Liu, G. Chen, and H. Wang, "Robust impulsive synchronization of uncertain dynamical networks," IEEE Trans. Circuits Syst.-I, vol. 52, no. 7, pp. 1431-1441, Jul. 2005.

[15] J. Lu, D. W. C. Ho, and J. Kurths, "Consensus over directed static networks with arbitrary communication delays," Phys. Rev. E, vol. 80, no. 6, pp. 066121-1-066121-7, Dec. 2009.

[16] T. Yang, Impulsive Systems and Control: Theory and Application. Commack, NY: Nova, 2001.

[17] J. Zhou, L. Xiang, and Z. Liu, "Synchronization in complex delayed dynamical networks with impulsive effects," Phys. A: Stat. Mech. Appl. vol. 384, no. 2, pp. 684-692, Oct. 2007.

[18] I. Fischer, Y. Liu, and P. Davis, "Synchronization of chaotic semiconductor laser dynamics on subnanosecond time scales and its potential for chaos communication," Phys. Rev. A, vol. 62, no. 1, pp. 011801-1011801-4, Jun. 2000.

[19] W. Lu and T. Chen, "New approach to synchronization analysis of linearly coupled ordinary differential systems," Phys. D, vol. 213, no. 2, pp. 214-230, Jan. 2006

[20] Z. Yang and D. Xu, "Stability analysis of delay neural networks with impulsive effects," IEEE Trans. Circuits Syst.-II, vol. 52, no. 8, pp. 517521, Aug. 2005.

[21] D. Bainov and P. Simeonov, Systems with Impulsive Effect: Stability Theory and Applications. Chichester, West Sussex, U.K.: Ellis Horwood, 1989.

[22] G. Zhang, Z. Liu, and Z. Ma, "Synchronization of complex dynamical networks via impulsive control," Chaos, vol. 17, no. 4, pp. 043126-1043126-9, Dec. 2007.

[23] Z.-H. Guan, D. J. Hill, and X. Shen, "On hybrid impulsive and switching systems and application to nonlinear control," IEEE Trans. Autom. Control, vol. 50, no. 7, pp. 1058-1062, Jul. 2005.
[24] Z.-H. Guan, D. J. Hill, and J. Yao, "A hybrid impulsive and switching control strategy for synchronization of nonlinear systems and application to Chua's chaotic circuit," Int. J. Bifur. Chaos, vol. 16, no. 5, pp. 229238, 2006.

[25] H. Zhang, Z.-H. Guan, and D. W. C. Ho, "On synchronization of hybrid switching and impulsive networks," in Proc. 45th IEEE Conf. Decis. Control, San Diego, CA, Dec. 2006, pp. 2765-2770.

[26] P. Li, J. Cao, and Z. Wang, "Robust impulsive synchronization of coupled delayed neural networks with uncertainties," Phys. A, vol. 373, pp. 261-272, Jan. 2007.

[27] B. Liu and D. J. Hill, "Robust stability of complex impulsive dynamical systems," in Proc. 46th IEEE Conf. Decis. Control, New Orleans, LA, Dec. 2007, pp. 103-108.

[28] Y.-W. Wang, M. Yang, H. O. Wang, and Z.-H. Guan, "Robust stabilization of complex switched networks with parametric uncertainties and delays via impulsive control," IEEE Trans. Circuits Syst.-I, vol. 56, no. 9, pp. 2100-2108, Sep. 2009.

[29] F. C. Hoppensteadt and E. M. Izhikevich, "Pattern recognition via synchronization in phase-locked loop neural networks," IEEE Trans. Neural Netw., vol. 11, no. 3, pp. 734-738, May 2000.

[30] T. Pereira, M. S. Baptista, and J. Kurths, "Detecting phase synchronization by localized maps: Application to neural networks," Europhys. Lett., vol. 77, no. 4, pp. 40006-1-40006-5, Feb. 2007.

[31] J. P. Hespanha and A. S. Morse, "Stability of switched systems with average dwell-time," in Proc. 38th IEEE Conf. Decis. Control, vol. 3. Phoenix, AZ, Dec. 1999, pp. 2655-2660.

[32] D. Liberzon, Switching in Systems and Control. New York: SpringerVerlag, 2003.

[33] L. Zhang and H. Gao, "Asynchronously switched control of switched linear systems with average dwell time," Automatica, vol. 46, no. 5 , pp. 953-958, May 2010.

[34] W. Wu and T. Chen, "Global synchronization criteria of linearly coupled neural network systems with time-varying coupling," IEEE Trans. Neural Netw., vol. 19, no. 2, pp. 319-332, Feb. 2008.

[35] F. Chung, Spectral Graph Theory. Providence, RI: AMS, 1997.

[36] C. Wu, Synchronization in Complex Networks of Nonlinear Dynamical Systems. Singapore: World Scientific, 2007.

[37] C. Wu, "Synchronization in networks of nonlinear dynamical systems coupled via a directed graph," Nonlinearity, vol. 18, no. 3, pp. 10571064, 2005.

[38] R. Horn and C. Johnson, Matrix Analysis. Cambridge, U.K.: Cambridge Univ. Press, 1990

[39] A. Halaney, Differential Equations: Stability, Oscillations, Time Lags. New York: Academic, 1966.

[40] M. Gilli, "Strange attractors in delayed cellular networks," IEEE Trans. Circuits Syst.-I: Fundam. Theory Appl., vol. 40, no. 11, pp. 849-853, Nov. 1993.

[41] M. E. J. Newman and D. J. Watts, "Scaling and percolation in the smallworld network model," Phys. Rev. E, vol. 60, no. 6, pp. 7332-7342, Dec. 1999. 